\title{
Macrodistrofia Lipomatosa. Reporte de un Caso
}

\author{
FRANCISCO CAMMARATA-SCALISI ${ }^{1}$, JOSÉ GREGORIO CAMPAGNARO GEREMIA ${ }^{2}$ \\ GLORIA DA SILVA ${ }^{3}$, CARLA OLIVO CUESTA ${ }^{4}$ \\ 1. Profesor Asistente de la Unidad de Genética Médica. Vicepresidente de la Asociación Merideña para el Síndrome de Down \\ (AMESID). \\ 2. Médico Traumatólogo. Ortopedista Infantil. Cirujano de Pie. Profesor Asociado Universidad de Los Andes. \\ 3. Médico Genetista. Profesor Instructor de la Unidad de Genética Médica. Departamento de Puericultura y Pediatría. \\ Universidad de Los Andes. \\ 4. Pediatría Puericultor. \\ Mérida-Venezuela.
}

\begin{abstract}
Macrodystrophia Lipomatosa. A Case Report

Macrodystrophia lipomatosa (ML), is a rare cause of non-hereditary localized gigantism, due to the progressive proliferation of mesenchymal tissue, with a disproportionate increase of fibroadipose tissue in one or more digits of the affected extremity. This congenital abnormality occurs more frequently in the distribution of the median nerve (arm) and plantar nerve (leg). Etiology is uncertain, and it is frequently misdiagnosed as another cause of macrodactylia. This paper reports a case of a 6 y.o. boy who presented overgrowth of the 1 st and 2 nd digit of the right foot, and slight increase of the $3 \mathrm{rd}$ digit in the other foot. A multidisciplinary evaluation was performed, corresponding to the second case reported in Venezuela.
\end{abstract}

(Key words: Macrodystrophia lipomatosa, localized gigantism, clinic presentation, etiology).

Rev Chil Pediatr 2010; 81 (6): 531-535

\section{RESUMEN}

La Macrodistrofia lipomatosa (ML), es una causa infrecuente de gigantismo localizado, no hereditario caracterizado por proliferación progresiva de todos los elementos mesenquimáticos, con un incremento desproporcionado en el tejido fibroadiposo en uno o más dedos de la extremidad afectada. Esta anormalidad congénita ocurre más frecuentemente en la distribución del nervio mediano en el miembro superior y nervio plantar en el miembro inferior. La etiología de la ML es incierta y es una entidad clínica que con frecuencia es diagnosticada erróneamente como otras formas de macrodactilia. Reportamos el caso de un escolar de 6 años de edad quien presentó sobrecremiento de $1^{\circ}$ y $2^{\circ}$ ortejo del pie derecho y leve aumento del $3^{\circ}$ ortejo del pie contralateral. Esta paciente fue evaluada de forma multidisciplinaria en nuestra institución y corresponde al segundo caso de ML reportado en Venezuela.

(Palabras clave: Macrodistrofia Lipomatosa, gigantismo localizado, presentación clínica, etiología).

Rev Chil Pediatr 2010; 81 (6): 531-535

Trabajo recibido el 05 de agosto de 2009, devuelto para corregir el 22 de septiembre de 2009, segunda versión el 06 de julio de 2010, aceptado para publicación el 02 de noviembre de 2010.

Correspondencia a:

Francisco Cammarata-Scalisi

E-mail: francocammarata19@gmail.com 


\section{Introducción}

La Macrodistrofia lipomatosa (ML) es una entidad infrecuente que causa gigantismo localizado congénito, no hereditario, con afectación de los dedos a través de la proliferación del tejido fibro-adiposo ${ }^{1}$. Este término fue empleado por primera vez por Feriz en 1925, para describir inicialmente el crecimiento unilateral del miembro inferior ${ }^{2}$, en 1996 se aceptó esta designación para las alteraciones en miembro superior $^{3}$. A pesar que la ML es de etiología incierta, diversas hipótesis incluyen: alteración de células somáticas durante el desarrollo, alteraciones en la circulación fetal ${ }^{4,5}$ y disfunción de nervios autonómicos ${ }^{1}$. En Venezuela existe sólo un caso publicado de $\mathrm{ML}$ en $3^{\circ}$ dedo de mano izquierda, en preescolar masculino, natural y procedente del Estado Nueva Esparta, región insular de nuestro país ${ }^{6}$. En el siguiente informe presentamos un nuevo caso de ML en una escolar procedente del Estado Barinas, valorada de forma multidisciplinaria en el Instituto Autónomo Universitario de Los Andes (I.A.H.U.L.A), que corresponde al segundo caso reportado de ML en Venezuela y el primero con afectación en miembro inferior.

\section{Caso clínico}

Escolar de sexo femenino de 6 años de edad, referida para su evaluación en la Unidad de Genética Médica-Universidad de Los Andes, por presentar hipertrofia en $1^{\circ}$ y $2^{\circ}$ ortejo en miembro inferior derecho.

Antecedentes familiares: Producto de unión consanguínea (primos hermanos), padre 47 años, madre 44 años, XI gestas, X paras, I aborto (no estudiado). De la unión de sus padres, la paciente es la mayor de una prole de cuatro hermanos. No se presenta caso similar en ambas familias.

Antecedentes personales: Producto de madre de 37 años, embarazo simple controlado, complicado con hiperémesis gravídica. Parto por vía vaginal a las 40 semanas de gestación, presentación cefálica. Peso al nacer: $3,3 \mathrm{~kg}$., talla al nacer: $48 \mathrm{~cm}$, sin patología en período neonatal. Adecuado desarrollo psicomotor.
Presentó aumento de volumen del $1^{\circ}$ y $2^{\circ}$ ortejos del pie derecho desde el nacimiento, manteniéndose en el tiempo y en igual proporción con respecto a los otros dedos del pie. No presenta antecedentes de trauma; marcha normal, sin embargo, emplea calzado de talla superior.

Examen físico: Peso: $16,1 \mathrm{~kg}(\mathrm{p} 3-10)$ y talla: $112 \mathrm{~cm}$ (p10-50). Desnutrición en rango crítico, normocéfala, cabello con signo de la bandera presente; hiperpigmentación infraorbitaria, dismorfias faciales menores caracterizadas por hipoplasia facial, narinas antevertidas, punta bulbosa, pabellones auriculares rotados posteriormente. Boca: malaoclusión dentaria, caries dental, paladar ojival. Cuello normal, tórax simétrico, cardiopulmonar: sin alteraciones. En región posterior de tórax izquierdo se evidencia hemangioma cavernoso de 3,1 x 2,3 centímetros. Región abdominal y área genital dentro de la normalidad. Extremidades: hemihipertrofia en miembro inferior derecho con perímetro superior en 2 centímetros, a nivel de músculo gastronemio con respecto al contralateral; hipertrofia del $1^{\circ}$ y $2^{\circ}$ ortejos del pie derecho, con diastasis entre ellos, (figura 1) y leve aumento de tamaño del $3^{\circ}$ ortejo en pie izquierdo.

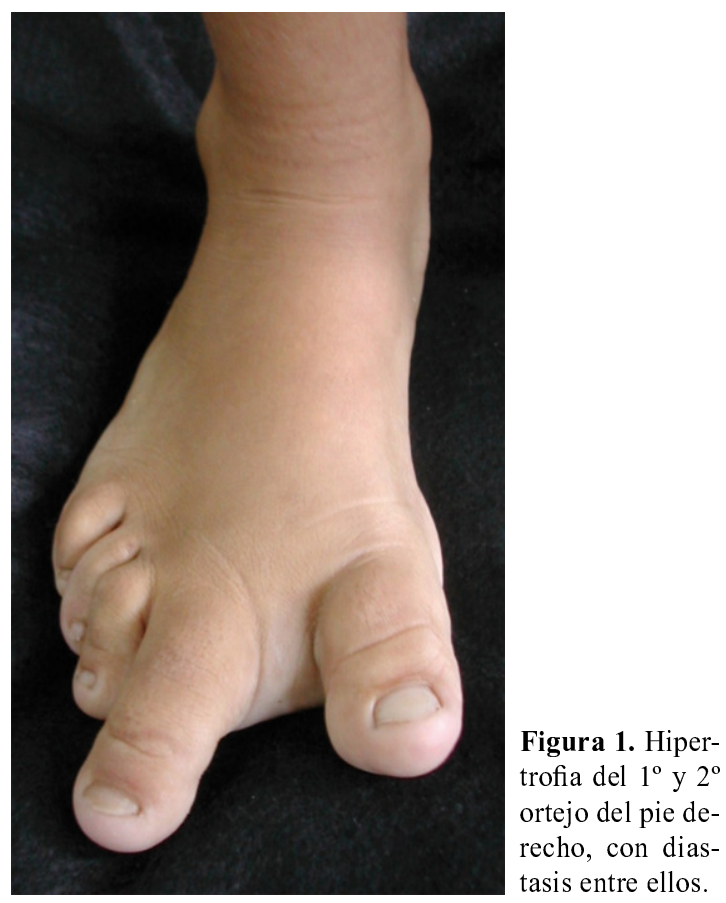


Exámenes de laboratorio dentro de la normalidad. Radiografía simple de pie derecho mostró aumento en el tejido blando, longitud y grosor en $1^{\circ}$ y $2^{\circ}$ metatarsiano con sus respectivas falanges (figura 2). Ultrasonido doppler venoso y arterial de miembros inferiores, sin evidencia de obstrucción del sistema venoso profundo, flujo fásico con la respiración, colapsables con maniobras de compresión, el cual es competente, así como el venoso superficial. Sistema venoso perforante bilateral suficiente y arterial en miembro inferior derecho con flujo de tipo trifásico. Señales presentes en arterias pedias e interdigitales.

\section{Discusión}

El gigantismo localizado congénito es una malformación infrecuente y puede ser parte de distintas entidades clínicas en la cuales hay sobreposición de signología. Para precisar su diagnóstico sindromático es necesario evaluar antecedentes familiares, manifestaciones cutáneas, sistémicas, estudios de imagen e histopatológicos ${ }^{7}$. La ML es una malformación infrecuente, cuya incidencia no ha sido repor$\operatorname{tada}^{6}$, congénita y no hereditaria de gigantismo localizado, caracterizado por incremento de todos los elementos mesenquemáticos ${ }^{8,9}$, comprometiendo uno o más dedos de la extremidad afectada ${ }^{8}$. Las áreas de predilección son los segmentos inervados por los nervios mediano y plantar ${ }^{9}$. Se presenta de forma indistinta en ambos sexos, sin embargo, algunos autores han reportado mayor frecuencia en el sexo masculino ${ }^{6}$. Es casi siempre unilateral y se presenta más frecuentemente en el miembro inferior. Los dedos más afectados son el $2^{\circ}$ y $3^{\circ}$ dedos y el menos es el $5^{\text {o10 }}$, infrecuentemente implica toda la extremidad ${ }^{11,12}$. El crecimiento del tejido blando es marcado en la parte distal y cesa en la pubertad ${ }^{10}$. En la paciente presentada en este informe se encuentran afectados el $1^{\circ}$ y $2^{\circ}$ ortejos del pie derecho con leve aumento de tamaño del $3^{\circ}$ ortejo en el pie contralateral, por lo que puede corresponder a un caso bilateral.

Los estudios de radiología simple presentan hipertrofia del tejido blando y a nivel óseo

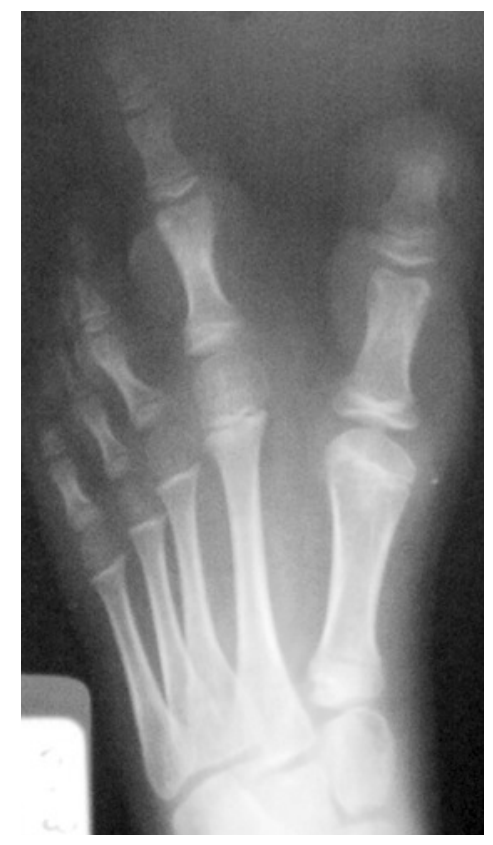

Figura 2. Radiografía simple: aumento en el tejido blando, longitud y grosor en $1^{\circ}$ y $2^{\circ}$ metatarsiano y sus falanges.

crecimiento excesivo similar a exóstosis. Las imágenes de resonancia magnética nuclear muestran un exceso de proliferación de tejido adiposo no encapsulado e infiltración grasa a nivel muscular ${ }^{13}$. Sone et $\mathrm{al}^{14}$, describieron los hallazgos de tomografía axial computarizada y resonancia magnética en dos pacientes con ML que demuestra la proliferación de tejido graso en el territorio del nervio mediano en mano y antebrazo. Por su parte, Bailey et $\mathrm{al}^{15}$, describen en tres pacientes, el exceso de tejido fibroadiposo en el área del nervio plantar de los pies. Los hallazgos patológicos se caracterizan por hipertrofia e infiltración de adipocitos a nivel de tejido celular subcutáneo, vaina de los nervios y periostio. Se diagnostican usualmente en la infancia ${ }^{16}$, sin embargo, para ello debe conocerse las diversas entidades que cursan con gigantismo localizado para poder establecer los posibles diagnósticos diferenciales.

Poco más de una centena de casos de ML han sido reportados en la literatura mundial. De etiología desconocida, entre las teorías de su origen se encuentran una degeneración lipomatosa, alteraciones en la circulación fetal, errores en la segmentación durante el desarrollo intrauterino, hipertrofia de la rama del nervio de la zona afectada y otras alteraciones in- 
trauterinas ${ }^{3}$. Van der Meer y $\operatorname{col}^{1}$, describen en Holanda ocho pacientes con ML confirmados por estudios histológicos, junto con otros 79 casos reportados en la literatura mundial. Mahafza y $\operatorname{col}^{17}$, describen la inusual asociación de sindactilia y ML localizada en mano. Existiendo descritas complicaciones en pacientes con ML. Ranawat y col $^{18}$, Coelho ${ }^{3}$, presentan casos en los cuales se produce síndrome de túnel carpiano como manifestación reumatológica en pacientes con ML. Meyer y Röricht ${ }^{19}$. reportan un caso de hamartoma fibrolipomatoso del nervio ulnar proximal asociado con ML, como causa inusual de síndrome de túnel cubital. Kozanoglu y $\mathrm{col}^{8}$, reportan síndrome de túnel tarsal, compresiones neurovasculares que fueron descritas en pacientes adultos, sin embargo, pueden presentarse en la adolescencia ${ }^{10}$. Aydos et $\mathrm{al}^{20}$, reportan el primer caso de asociación de ML y lipomas subcutáneos en una lactante mayor de sexo femenino de 16 meses de edad. Finalmente, Fritz y Swischuk ${ }^{21}$, reportan un particular caso de ML en un paciente pediátrico que no sólo presentó la alteración típica a nivel de extremidades sino extensión abdominal de tejido adiposo hipertrófico. En la paciente reportada en este informe se presentó leve hemihipertrofia en miembro inferior afectado; sin embargo, no se presentó extensión del defecto a nivel abdominal.

El sobrecrecimiento localizado del tejido blando y óseo puede deberse a distintas etiologías y varían en severidad, afectando desde un dedo hasta la mitad del cuerpo. Las enfermedades o Síndromes malformativos con los cuales hay que realizar diagnóstico diferencial de la ML incluyen: síndrome de Kippel-TrenaunayWeber, síndrome de Proteus, neurofibromatosis tipo $1^{22,23}$, hamartoma fibrolipomatoso ${ }^{22}$, síndrome de Maffucci ${ }^{22,23}$, enfermedad de Ollier ${ }^{23}$, enfermedad de Milroy, síndrome de BannayanRiley-Ruvalcaba ${ }^{22}$, displasia fibrosa poliostótica, síndrome de Temtamy, hemihipertrofia aislada $^{23}$, linfedema crónico, malformaciones linfangiomatosas. La paciente inicialmente fue valorada en otro centro y referida a la Unidad de Genética Médica-Universidad de Los Andes y al Servicio de Ortopedia y Traumatología del I.A.H.U.L.A., con el diagnóstico presuntivo de síndrome de Kippel-Trenaunay-Weber. Poste- rior a la realización del ultrasonido doppler venoso y arterial de miembros inferiores, el cual no reportó anormalidad, y por su curso y características clínicas se planteó el diagnóstico de ML.

El tratamiento es quirúrgico (acortamiento y amputación) y debe realizarse posteriormente en la pubertad, por ello el seguimiento multidisciplinario de su evolución fenotípica es indispensable.

La ML es una entidad benigna pero con implicaciones estéticas. Ante una patología poco conocida y sin incidencia reportada, es necesario establecer los distintos diagnósticos diferenciales anteriormente comentados con el objeto de reconocer cada una de estas entidades de gigantismo localizado. Los aspectos clínicos, estudios complementarios y de ser posible estudio molecular son necesarios para establecer el diagnóstico definitivo, y con ello proporcionar un adecuado tratamiento e impartir un oportuno consejo genético al grupo familiar, a pesar que la ML no se trate de una entidad hereditaria. La etiología sigue siendo tema de investigación, además en este informe describimos algunas patologías asociadas y complicaciones que pueden presentarse en su curso clínico. Hasta el momento el caso presentado en este informe corresponde el segundo de ML presentado en Venezuela, el primero presente en miembro inferior y el único evaluado en la Unidad de Genética Médica-Universidad de Los Andes, centro de referencia del occidente del país, en los últimos cinco años.

\section{Referencias}

1.- $\quad$ van der Meer S, Nicolai JP, Meek MF: Macrodystrophia lipomatosa: macrodactyly related to affected nerves, and a review of the literature. Handchir Mikrochir Plast Chir 2007; 39: 414-7.

2.- Feriz H: Makrodystrophia Lipomatosa progressiva. Virchows Arch 1925; 260: 308-68.

3.- Coelho PC: Macrodystrophia lipomatosa. A rare disease with rheumatic manifestations. Acta Reumatol Port 2007; 32: 61-5.

4.- Singla V, Virmani V, Tuli P, Singh P, Khandelwal N: Case Report: Macrodystrophia lipomatosa - Illustration of two cases. Indian J Radiol Imaging 2008; 18: 298-301. 
5.- Gupta SK, Sharma OP, Sharma SV, Sood B, Gupta S: Macrodystrophia lipomatosa: Radiographic observations. Br J Radiol 1992; 65: 769-73.

6.- Sifontes K, López L, Rodríguez C, Hernández R: Macrodistrofia lipomatosa. Caso Clínico. Informed 2009; 11: 261-4.

7.- Wahab S, Khan RA, Ahmad I: Congenital localized limb hypertrophy: macrodystrophia lipomatosa. JBR-BTR 2008; 91: 209-10.

8.- Kozanoglu E, Koc F, Goncu K: Macrodystrophia lipomatosa with multiple entrapment neuropathies: a case report. Int J Neurosci 2008; 118: 545-53.

9.- D 'Costa GF, Taksande RV, Pandya BS, Najmi S, Dua $S$, Patil YV: Macrodystrophia lipomatosa: a case report. Indian J Pathol Microbiol 2007; 50: 572-4.

10.- Hafeez S, Syed M, Syed A: Macrodystrophia lipomatosa. JK-Practitioner 2006; 13: 41-2.

11.- Oztürk A, Baktiroğlu L, Oztürk E, Yazgan P: Macrodystrophia lipomatosa: a case report. Acta Orthop Traumatol Turc 2004; 38: 220-3.

12.- Bansal VP, Harmit S: Monomelic macrodystrophia lipomatosa. A case report. Int Orthop 1989; 13: 77-9.

13.- Pandey $A K$ : Magnetic resonance imaging of a case of monomelic macrodystrophia lipomatosa. Australas Radiol 2007; 51 Suppl: B227-30.

14.- Sone M, Ehara S, Tamakawa Y, Nishida J, Honjoh S: Macrodystrophia lipomatosa: CT and MR findings. Radiat Med 2000; 18: 129-32.
15.- Bailey EJ, Thompson FM, Bohne W, Dyal C: Macrodystrophia lipomatosa of the foot: a report of three cases and literature review. Foot Ankle Int 1997; 18: 89-93.

16.- Tuzuner T, Parlak AH, Kavak A, Alper M: A neglected case of macrodystrophia lipomatosa of the foot in an elderly man. J Am Pediatr Med Assoc 2005; 95: 486-90.

17.- Mahafza WS, Haroun AA, Abdul-Wahab AD, Hadidi $S T$ : Macrodystrophia lipomatosa with syndactyly. Saudi Med J 2008; 29: 1194-6.

18.- Ranawat CS, Arora MM, Singh RG: Macrodystrophia lipomatosa with carpal-tunnel syndrome. A case report. J Bone Joint Surg Am 1968; 50: 1242-4.

19.- Meyer BU, Röricht $S$ : Fibrolipomatous hamartoma of the proximal ulnar nerve associated with macrodactyly and macrodystrophia lipomatosa as an unusual cause of cubital tunnel syndrome. J Neurol Neurosurg Psychiatry 1997; 63: 808-10.

20.- Aydos SE, Fitoz S, Bökesoy I: Macrodystrophia lipomatosa of the feet and subcutaneous lipomas. Am J Med Genet A 2003; 119A: 63-5.

21.- Fritz TR, Swischuk LE: Macrodystrophia lipomatosa extending into the upper abdomen. Pediatr Radiol 2007; 37: $1275-7$.

22.- Levine $C$ : The imaging of body asymmetry and hemihypertrophy. Crit Rev Diagn Imaging 1990;31:1-80.

23.- Navarro A, Sanchis A, Escobar M, Llorens R: Macrodactilia aislada en recién nacido. An Pediatr 2009; 71 : 459-69. 